\section{Mehr Sicherheit, weniger Verschleiß}

Das Mess- und Aufbereitungssystem DentaPort ZX des Herstellers Morita ist seit Kurzem in der 3. Gerätegeneration verfügbar und zeichnet sich durch die neue Sicherheitsfunktion OTR (Optimum Torque Reverse) aus. Diese bietet eine höhere Sicherheit im Rahmen der endodontischen Therapie: Durch die permanente Kontrolle des Drehmoments während der Aufbereitung - für OTR wird messtechnisch nur eine sehr kleine Winkeldrehung der Feile benötigt - reduziert sich das Risiko eines Feilenbruchs auf ein Minimum. Wie die folgenden Ausführungen darlegen, erhöhen weitere Automatik- und Sicherheitsfunktionen die endodontische Arbeitssicherheit.

Im Rahmen der Wurzelkanalaufbereitung werden hohe Anforderungen an den Behandler und dessen Instrumente gestellt: Sie reichen von der notwendigen vollständigen Bearbeitung der Wurzelkanalwand über den Erhalt der apikalen Konstriktion, dem Vermeiden einer Überstopfung des Debris bis hin zum Sicherstellen eines möglichst geringen Bruchrisikos der Instrumente, um nur einige zu nennen. Denn ebenso wie die Via falsa ist die Fraktur eines Instruments im Wurzelkanal sicherlich eines der unwillkommensten Ereignisse im Laufe der endodontischen Therapie. Nicht umsonst wurden aufgrund der Anforderungen an die Instrumente zur Wurzelkanalaufbereitung bereits 1974 standardisierte ISO-Normen festgelegt und immer wieder erweitert (DIN EN ISO 3630-2: 2013-08). Auch die (Weiter-)Entwicklungen von Feilen und endodontischen Antriebsmotoren orientieren sich an der Arbeitssicherheit. Seit festgestellt wurde, dass die Stärke der Kanalkrümmung, die Drehzahl während der Aufbereitung und das Drehmoment Einfluss auf das Risiko des Feilenbruchs haben (Gambarini G. Endod Dent Traumatol, 2000; 16: 95-100; Gambarini G. J Endod. 2001; 27: 772-774), werden maschinelle Endodontiesysteme mit (variabler) Drehmomentbegrenzung angeboten. Das ist deshalb wichtig, weil die Feile durch die Rotation im Kanal stark beansprucht wird. Damit wirken auf die Feile permanent mechanische Einflüsse, wie beispielsweise der Reibwiderstand. Dieser tritt bei der rotierenden Aufbereitung als Drehmoment auf, wodurch sich die Feile verwindet und frakturieren kann.

Warum bietet OTR mehr Sicherheit? Moderne Aufbereitungsmotoren arbeiten mit der Standard-Torque-Reverse-Funktion: Dabei dreht sich die Feile automatisch bei starker Beanspruchung gegen den Uhrzeigersinn und senkt so das Risiko einer Instrumentenfraktur. Dazu wird das auf die Feile wirkende Drehmoment automatisch gemessen. Bei zu starker Belastung dreht sich die Feile automatisch $90^{\circ}$ zurück. Die im DentaPort ZX angebotene OTR-Funktion unterscheidet sich im Vergleich zu den bisher angebotenen Geräten: Sie benötigt nur eine relativ kleine Winkeldrehung der Feile, um die aktuelle Belastung automatisch zu messen. Auf diese Weise arbeitet der Zahnarzt mit optimierten Drehwinkeln in Schneid- wie auch in Rückdrehrichtung der Feile. Dadurch ist, bei Nickeltitanfeilen ohne Torsions- und somit ohne erhöhte Frakturgefahr, eine Schneidrichtung von einer halben Umdrehung $\left(180^{\circ}\right)$ sowie eine Vierteldrehung $\left(90^{\circ}\right)$ gegen die Schneidrichtung möglich. Auch bei der Rückdrehung misst das System das Drehmoment automatisch: Ist das Drehmoment nach der $90^{\circ}$-Rückdrehung immer noch $\mathrm{zu}$ hoch, dreht sich die Feile einmalig über die $90^{\circ}$ hinaus weiter entgegen der Schneidrichtung, bis sie sich wieder in einem sicheren Zustand befindet.

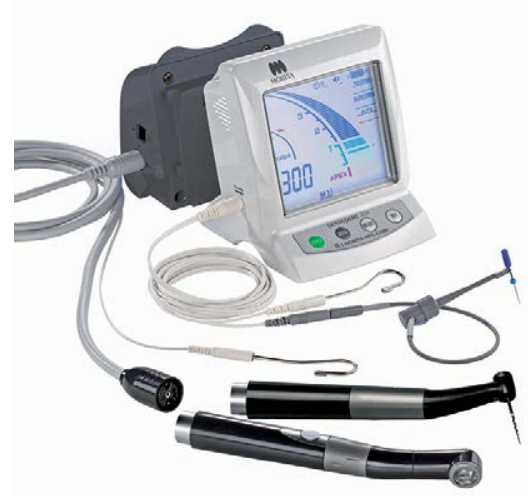

Das neue Prinzip sorgt für eine hohe Schneideeffektivität. Die Schneidleistung geht in der $180^{\circ}$-Umdrehung erst dann in „Torque Reverse“, wenn das voreingestellte Drehmoment erreicht wird - andernfalls arbeitet die Feile kontinuierlich in Schneidrichtung weiter. Zudem kommt es mit vergleichsweise geringen Antriebs- momenten aus, was wiederum Belastung und Verschleiß der Feilen deutlich reduziert. Untersuchungen des Herstellers zufolge hat die Drehzahl maßgeblichen Einfluss auf die Frakturanfälligkeit von Nickeltitanfeilen: Je kleiner die Drehzahl, desto geringer die Frakturgefährdung (Dietz DB et al. J Endod. 2000; 26: 68-71). OTR arbeitet mit 100-500 U/min (in 3 Stufen) - die Standardfunktion Auto-TorqueReverse bei Morita-Geräten dagegen mit 150-800 U/min (in 8 Stufen). Die Behandlungssicherheit wird durch eine StartStopp-Funktion zusätzlich erhöht, welche die Rotation beim Herausnehmen der Feile automatisch stoppt.

Gerätestandard optimiert Sicherheit Für weitere Sicherheiten sorgen die zusätzlichen Betriebsfunktionen des DentaPort ZX Set OTR: Die neue Gerätegeneration wurde mit einer internen Feilenelektrode ausgestattet, die der Zahnarzt bei Bedarf austauschen kann. Sollte dies erforderlich sein, meldet sich das Gerät selbstständig mit einem akustischen Hinweis. Auch einer weiteren Funktion kommt eine wichtige Rolle zu: Da die zunehmende Betriebsdauer die Genauigkeit der Torque-Kontrolle beeinflussen kann, sorgt eine regelmäßige Kalibrierung für gleichbleibende Qualität und Funktionalität - dies bietet dem Anwender ein zuverlässiges Instrument, das dauerhaft ein präzises Arbeiten ermöglicht.

\section{Fazit}

Moderne maschinelle Aufbereitungssysteme bieten dem endodontischen Behandler eine höhere Sicherheit auf dem Weg zu einer erfolgreichen Therapie. Die neu auf dem Markt erhältliche Optimum Torque Reverse-Funktion in Moritas modularem Mess- und Aufbereitungssystem DentaPort ZX Set OTR sorgt u. a. mit einer verbesserten automatischen Messung des Drehmoments und einer Reduzierung der Drehzahl zur Vermeidung von Feilenbruch für zusätzliche Sicherheit - somit profitiert der Zahnarzt auch von einer erhöhten Effizienz und Wirtschaftlichkeit im Rahmen der Therapie.

\section{Kontakt}

Morita Europe GmbH

Justus-von-Liebig-Straße 27a

63128 Dietzenbach

info@morita.de

www.morita.com/europe

Dieser Beitrag entstand mit freundlicher Unterstützung der J. Morita Europe $\mathrm{GmbH}$, Dietzenbach. 